\title{
CIUDADES Y PERIFERIAS SOSTENIBLES. Una revisión de conceptos y enfoques aplicados a España y Castilla y León
}

\author{
Lorenzo LÓPEZ TRIGAL y Carlos Emilio RELEA FERNÁNDEZ \\ Departamento de Geografía. Universidad de León
}

\section{CONCEPTOS PREVIOS.}

El concepto del desarrollo sostenible apareció recogido por primera vez en 1987, como argumento central del Informe de Naciones Unidas, Our Common Future, en el que aparecían recogidas las preocupaciones de décadas anteriores en la literatura económico-ambiental iniciadas en la Conferencia de Estocolmo de 1972, escenario donde se elaboró el primer discurso ecologista tecnocrático. Conceptos como "conservación" o "ecodesarrollo" fueron pasando al olvido debido a la progresiva generalización y profusión de uso del nuevo concepto, nunca antes experimentada y que lo ha llevado a ser adoptado por colectivos muy dispares en su ideología. Esta situación se ha visto favorecida por la vaguedad y ambigüedad inherente al propio concepto, hecho que ha provocado la formulación de más de doscientas definiciones de desarrollo sostenible en las que se enfatizan los valores y prioridades de los diferentes agentes que las promueven (REDCLIFF, 2000: 70). Pero, a pesar de esta proliferación de definiciones, se puede percibir, a grandes rasgos, el debate existente en la división de planteamientos entre los "desarrollistas" defensores de una "sostenibilidad débil" y, dominada por un enfoque económico y por lo tanto más permisiva con las actuaciones humanas en su relación con la naturaleza, y los "ambientalistas", entre los que abogan por una "sostenibilidad fuerte", donde predomina un enfoque ecológico basado en las condiciones de incertidumbre, ignorancia e irreversibilidad y que establece unas relaciones mucho más restrictivas.

"Las ciudades son el principal lugar de producción, consumo y creatividad civilizada, así como la fuente de muchos daños ambientales y el lugar en el que se manifiestan con mayor virulencia. Muchos de los problemas que surgen se deben a las características particulares de las ciudades y de la vida urbana y, viceversa, muchas soluciones son específicas de las ciudades y de la gestión urba- 
na" (COMISIÓN EUROPEA, 1996). Por ello no es extraño que una de las importantes aportaciones que tendrá este nuevo enfoque sea su aplicación al estudio y comprensión del fenómeno urbano que se está descubriendo como uno de los principales escenarios en el que buscar y experimentar nuevas alternativas para lograr un desarrollo sostenible. La búsqueda de la ciudad sostenible tendrá una especial significación a partir de 1990, especialmente cuando se comience a poner de manifiesto la no sostenibilidad de la ciudad difusa derivada de las actuales tendencias de suburbanización y de formación de conurbaciones en las que se incrementan los problemas derivados de la ciudad sobre su entorno y sobre las propias condiciones de vida de sus habitantes.

Como prueba de la difusión de este nuevo concepto de sostenibilidad está el hecho de que en el seno de la Unión Europea y de la OCDE, de los problemas de crecimiento y de declive de las ciudades que provocaron el interés del estudio de las ciudades en las décadas de los 70 y 80 se haya pasado al interés por el fomento del buen gobierno en la ciudad, atendiendo a aspectos relacionados con el planeamiento, la calidad de vida, el control de impactos, la optimización de infraestructuras, el diseño de modelos urbanos alternativos, la regeneración urbana y remodelación de barrios desfavorecidos.

En la actualidad este nuevo enfoque de la sostenibilidad ha alterado la visión y estudio geográfico del fenómeno urbano. Pero antes de incidir más en el mismo, se hace conveniente revisar con antelación, conceptos y enfoques en torno a ciudad y periferia urbana, que nos parecen claves en el presente análisis.

Desde un enfoque locacional el concepto de ciudad es el de "centro en un plano espacial" (Manuel de Terán), en cuanto que las actividades de una ciudad no tienen sentido en sí mismas sino se traducen geográficamente en una ordenación espacial de los diferentes usos residenciales, comerciales, industriales, administrativos o de otro tipo del suelo urbano, lo que impone, en consecuencia, la necesidad de considerar el significado específico de la noción de centralidad como elemento decisivo de la organización de los espacios urbanos. Desde estos presupuestos, se define la noción de centro como "nudo de relaciones y de comunicaciones y polo de integración, coordinador y de decisión", fielmente representado en el CBD o centro de negocios de la gran ciudad norteamericana, que suma a la vez la city que ejerce la función financiera y la cité para la función comercial, y en ocasiones, en la ciudad europea, puede que coincida el centro histórico y simbólico de la ciudad con el centro comercial y de negocios. 
A partir de este centro se distribuirán sucesivamente las diferentes aureolas que delimitan los usos del suelo urbano, tomando como base elementos económicos o sociológicos que van por lo general de más a menos valores, como en el caso del precio del suelo, la distancia al centro, las diferencias de densidad de usos, las distintas intensidades de tráfico y, en algunos casos, las preferencias residenciales de la población urbana o la especialización comercial. La ciudad es concebida, de esta manera, como un centro económico y demográfico capaz de consolidarse como un factor decisivo en la organización del espacio o centro polarizador en torno al cual se articula un territorio en el doble sentido intraurbano y extraurbano.

En esta línea, el esquema histórico del centro principal de la ciudad europea o americana a lo largo del siglo XX ha pasado por diferentes fases: primero, el centro tiende a agrandarse y a monopolizar las funciones centrales; segundo, tiende a dispersarse en centros secundarios por el extrarradio de la ciudad manteniéndose a la vez el centro principal; tercero, este centro responde ante la situación de mudanza de tres maneras, bien sobrevive renovándose y modernizándose (en forma de sustitución de edificios puntuales manteniendo el viejo viario y tejido urbano o en forma de renovación por entero con unos volúmenes de ocupación más intensos), bien prevalece alguno de los centros subsidiarios de orden inferior imponiéndose al viejo centro, o bien se crea uno o más centros en los nuevos espacios de la periferia con una mejor accesibilidad triunfando de momento sobre los viejos y menos viejos centros de los espacios centrales de la ciudad, originándose en ocasiones en las metrópolis situaciones de policentralidad principal. En este sentido, en algunas de las ciudades de mayor talla, la delimitación del centro y periferia ya no es tan simple y no se puede diferenciar fácilmente entre el polo urbano y la corona periurbana, a la vez que en general en las ciudades de talla mediana y grande aparece un marcado espacio de transición entre el centro y la periferia, el de los espacios pericentrales, que en realidad son los barrios periféricos más próximos al centro o centros de la ciudad.

Este esquema se puede observar, en una cierta medida, en ciudades de la región de Castilla y León. El análisis de las ciudades de Valladolid o León en cuanto a la evolución del centro tradicional y la consolidación de nuevos centros ante los problemas de adaptación edificatoria, de accesibilidad, de suficiencia de nuevas dotaciones y espacios para la circulación de los viejos centros así lo demuestra. En otras ciudades, como es el caso de Salamanca, la ciudad histórica ha podido adaptarse por ahora a las necesidades de un centro funcional mo- 
derno.

\section{LA CIUDAD DE LA PERIFERIA.}

La dinámica de las ciudades en el tiempo ha producido numerosos cambios en la estructuración interna del espacio urbano y el papel del centro y de la periferia, cada vez más extensa y densa demográfica y funcionalmente en el conjunto de las áreas urbanas y metropolitanas, hasta el punto que en las ciudades donde los suburbios y núcleos periurbanos han adquirido una mayor desenvoltura, parece que estos se desquitan de su papel anterior de dependencia respecto del centro urbano y pasan a desempeñar incluso funciones centrales por causa de desplazamientos de actividades terciarias de todo tipo. En efecto, en las ciudades medias y grandes en mayor medida, el espacio periférico se extiende y gana en población y funcionalidad y lo hace, por lo general, en detrimento de la pérdida correspondiente del centro de la ciudad y de los mismos espacios pericentrales, el de los arrabales y barrios periféricos, que en ciertos casos entran ahora en declive.

Por algún autor se ha afirmado que "la extensión del hecho urbano en el territorio exterior de la ciudad es, con toda seguridad, el acontecimiento más importante del urbanismo de las últimas décadas del siglo veinte y paulatinamente los entornos metropolitanos se convierten en una ciudad única, concebida a escala del automóvil y de los medios de comunicación rápidos" (TORRES I CAPELL, 1997: 66). En el modelo de ciudad norteamericana se han llegado a definir los suburbios de la periferia como "barrios residenciales en las afueras de las ciudades donde casi todo el mundo quiere vivir y no se es nadie sin su parcela de verde bien afeitado".

Claro que este afán se ha extendido también a la ciudad europea, en la que el entorno urbano no termina ya en los arrabales y barrios periféricos a extramuros y adosados a la vieja ciudad, pues más allá ha surgido una nueva periferia desde hace décadas, un nuevo extrarradio conocido como espacio periurbano y suburbano, con caracteres de interpenetración de lo rural y lo urbano, cuyos vínculos con el territorio rural o natural siguen siendo tan fuertes como en el pasado, apoyándose a menudo en viejos núcleos de población del alfoz medieval de la ciudad (León, Salamanca, Zamora, Segovia...), adonde emigran sectores de población de muy diferente renta y adonde se exportan los problemas ambientales generados en la ciudad confundiéndose en una mixtura cada vez más de espacio rururbano según avanza el proceso de extensión de la periferia. Las áreas pe- 
riurbanas son, en este sentido, nuevos y diferentes espacios de los propiamente urbanos o rurales, participando más de los primeros por su hábitat, su plurifuncionalidad y diversificación de actividades y sobre todo por ser el espacio realmente de reserva y urbanizable y el soporte de los amplios espacios de todo tipo que necesita la ciudad.

Muchas tipologías de periferias aparecen en realidad en nuestras ciudades medias y grandes: así, mientras en Madrid se constatan tres coronas en un radio de unos 50 kilómetros (pericentral, metropolitana y rururbana), en León se resumen en una zona de unos 10 a 12 kilómetros de radio en los ejes de mayor circulación, que se apoya en casi una centena de núcleos preexistentes o de nuevas urbanizaciones entre los que alternan espacios en degradación y abandono, eriales o vacíos periurbanos, a la espera de una mejor oportunidad especulativa con otros revalorizados por nuevas actividades no agrarias. En realidad, en las periferias urbanas hay una situación multidiversa de espacios en graduaciones y transiciones de toda clase que quedan interrumpidas por diferentes bordes y frenos de los espacios protegidos, mientras que las nuevas dotaciones e infraestructuras y los ejes viarios principales resultan sumamente atractivos. Todo ello origina un proceso de expansión física hecha "a saltos", dejando atrás espacios agrarios e intersticios de tipo rural, o "por paquetes" al pie de las áreas centrales, sin olvidar el papel decisivo de la variable administrativa y de la linde municipal, como, asimismo, el de "los promotores inmobiliarios que pueden recurrir, por distintas estrategias, a edificar primero más lejos y luego irse acercando a la ciudad central" (GRUPO ADUAR, 2000: 266). Este es el caso de la dirección del crecimiento del barrio de Gamonal en la periferia norte de Burgos.

\section{LA CIUDAD, DESDE UN ENFOQUE SOCIAL Y AMBIENTAL.}

La ciudad, desde un enfoque social y ambiental es resultado de la acción humana y del medio físico, un producto cultural y multiforme, a menudo prolongado en el tiempo, sobre un espacio a la vez próximo y lejano. Así, la ciudad es "un asentamiento denso y permanente de individuos socialmente heterogéneos" (Manuel de Terán), "la proyección sobre el terreno de la sociedad global con sus luchas, conflictos, tensiones" (Henry Lefebvre), o en otros términos "una organización destinada a maximizar la interacción social" (Paul Claval). Al mismo tiempo, la urbanización es ya un hecho irreversible que ha significado el soporte de la difusión de la humanización de la Tierra. "La ciudad ha sido un milagro a hechura del hombre. Sus muros eran los confines de la libertad y 
circundaban la única comunidad sin resabio tribal" (Salvador Giner). En este orden, la ciudad es esencialmente intercambio y comunicación entre sus pobladores, entre actividades e instituciones diferentes, y en el caso de la ciudad europea, a diferencia de otros modelos de ciudad, sus elementos definidores han sido "la mezcla de funciones, la referencia de la ciudad a la escala humana, la calidad y la estética de los espacios públicos” (BOFILL, 1998: 109).

Sin embargo, la ciudad europea "se encuentra hoy carcomida por diversos elementos que alteran su justificación histórica: la desintegración de la forma, sin la cual el ciudadano pierde referencias de vida colectiva; la presencia de guetos en los que se zonifican determinadas funciones; la dispersión en suburbios residenciales que implica una negación casi absoluta de la ciudad; el crecimiento caótico de una periferia densa, en la que las tipologías urbanas se organizan sin adecuarse a la permanencia morfológica de la ciudad" (ORIOL BOHIGAS, en El País, 12 enero 1995). Desde esta visión la ciudad periférica es más bien una serie de áreas urbanizadas fragmentadas e inconexas, aunque "algunas áreas adquieren o consolidan su calidad a través de la culminación de su propio proceso de urbanización o de la puesta en valor de factores de localización, otras, en cambio, acentúan su marginalidad, no acaban de entrar en el contexto de la modernidad, o se convierten en espacios ambiguos y susceptibles de transformación. Es el caso de gran parte de los espacios industriales y también de algunas áreas agrícolas periféricas" (TORRES I CAPELL, 1997: 66). "La ciudad, mientras tanto, se va vaciando de contenido, las relaciones vecinales, la regulación de comportamientos por conocimiento y afectividad, la identidad con el espacio, las probabilidades de contacto que ofrece el espacio público se van diluyendo. Los barrios, que son el terreno de juego donde se hace cotidiana la esencia de la ciudad, se eclipsan" (RUEDA, VARIOS AUTORES, 1998-1999).

De este modo, la ciudad actual está poco integrada con su medio circundante por lo que se hace necesario recomponer su urdimbre humana y limitar los efectos negativos que tal modelo de crecimiento del proceso de suburbanización tiene sobre el medio ambiente del entorno, en detrimento de los espacios rurales y naturales, pues la construcción de la ciudad actual no aumenta la probabilidad de contactos, la acumulación de informaciones y la accesibilidad a las mismas. Por el contrario, a resultas de ello es "una ciudad que se difumina en el campo ocupando áreas cada vez más extensas, la ciudad difusa que tiene todo disperso, separado, funcional (la universidad, la industria, la residencia, las áreas comerciales, las oficinas, etc. se separan físicamente) y segregado socialmente, unien- 
do las partes a través de una densa red de carreteras y vías segregadas de transporte privado, forma de proceder que multiplica en tres aspectos la no sostenibilidad de la ciudad difusa: el consumo del suelo, el consumo de energía y materiales y la tendencia a explotar y desestructurar los sistemas del entorno más allá de su capacidad de carga". En consecuencia, "lo importante en la ciudad difusa son las redes. Si vamos en coche o contactamos a través de los medios de comunicación, el espacio público no es relevante y la ciudad tampoco" (RUEDA, VARIOS AUTORES, 1998-1999). De esta manera podemos constatar que si la reducción del hacimaniento característico de las ciudades a finales del siglo XIX y principios del XX mejoró la salubridad urbana, la extrema dispersión actual de los usos y la gran dependencia del transporte, especialmente el privado, constituyen hoy uno de los principales factores de deterioro del medio ambiente urbano (NAREDO, 2000). Se defiende desde esta posición que el modelo que se acomoda mejor a los principios de sostenibilidad es el de la ciudad mediterránea compacta y densa con continuidad formal y multifuncional, que permite obtener una vida social cohesionada al mismo tiempo que se ahorra suelo, energía y recursos materiales y se preservan los sistemas agrícolas y naturales.

Pero, cabe cuestionar que este modelo es difícil observarlo hoy en día, por ejemplo, en ciudades andaluzas de distinto tamaño como Córdoba o VélezMálaga, caracterizadas hasta hace poco como ciudades compactas, en donde avanza también la extensión periférica, en parte también en forma difusa, en detrimento de los recursos de sus respectivas vegas. Por otro lado, la buena práctica de gestión del planeamiento en ciudades del norte de España ha tenido en este orden diferentes resultados: mientras que la ciudad de Vitoria se ha mantenido como ciudad compacta a pesar de su fuerte crecimiento reciente, un parecido crecimiento de la ciudad de Pamplona, controlado y bien ordenado en su municipio, ha engendrado en los términos limítrofes un salpicado de nuevas barriadas y urbanizaciones periurbanas que nos advierten de la presencia de dos diferentes modelos de ciudad, compacta y difusa, en la misma área urbana. "La idea genérica de ciudad mediterránea, como sinónimo de compacidad, densidades relativamente altas, mezcla de usos y diversidad, ya no encuentra una correspondencia clara con nuestras aglomeraciones urbanas y metropolitanas. Pues, sobre todo en las grandes ciudades, solamente el núcleo o núcleos centrales de las mismas conservan dichos atributos" (MONCLÚs, 1998).

Se ha consolidado así en España una ciudad distinta a la anterior, en cuanto que la urbanización difusa de la periferia de muchas de las ciudades medias y 
grandes anuncia la presencia más bien de áreas urbanas y áreas metropolitanas que se amplían y densifican a su vez y que suman a los espacios urbanos los nuevos espacios periurbanos y rururbanos de su entorno, consecuencia directa del desarrollo de las mallas de infraestructura y del nuevo modelo de ciudad dominante. "Dispersión territorial que provoca un crecimiento extraordinario del perímetro exterior de la urbanización, con el consiguiente aumento de los problemas de interrelación entre la ciudad y el territorio, y de la fricción entre áreas urbanas y el territorio agrícola, forestal o simplemente natural. Esta fricción o conflicto entre la ciudad y el territorio o el paisaje se convierte en concepto esencial del urbanismo de finales de este siglo" (TORRES I CAPELL, 1997: 67). En la raíz de todo ello está la situación de un medio ambiente modificado por los flujos de origen urbano (migraciones de población, flujos de agua, de energía, de productos alimenticios...) en todas sus componentes, físicas y humanas (PIGEON, 1994).

\section{EL URBANISMO SOSTENIBLE}

Volviendo al comienzo de este estudio, a raíz de los principios declarados en la Conferencia de Naciones Unidas sobre Medio Ambiente y Desarrollo Sostenible en Río de Janeiro, 1992, se desemboca en el nuevo urbanismo de defensa de una perspectiva integrada y a largo plazo de las cuestiones ambientales, socioculturales y económicas de cada ciudad. Se avanza así en el modo de entender la ciudad como un ecosistema, un asentamiento donde vivimos, trabajamos, nos relacionamos o simplemente visitamos, con una diversidad de gentes y de culturas y medios que puede aprovecharse para un desarrollo con más calidad de vida, servicios, infraestructuras, cultura y posibilidades, una ciudad en la que los ciudadanos son protagonistas y participantes de la transformación social y ecológica, concluyendo que la vida humana en nuestro planeta no puede ser sostenible sin unas comunidades locales viables que no limiten su desarrollo futuro. Este enfoque ecológico de la ciudad es el que ha dado pie a uno de los debates más interesantes en el urbanismo actual, protagonizado por aquellos que defienden la ciudad compacta, mediterránea, frente a la insostenibilidad de la ciudad difusa, sin que por ello debamos de aceptar que las ciudades compactas actuales sean de por sí sostenibles (Ver modelos compacto y difuso de ocupación del territorio en RUEDA, 2002: 24-25).

A lo largo de los años noventa, cada vez más gobiernos, administraciones públicas, partidos políticos y todo tipo de empresas y asociaciones han suscrito ta- 
les principios de sostenibilidad como un código ético de comportamiento a la hora de llevar a cabo sus actuaciones respectivas teniendo en cuenta las necesidades de las generaciones futuras, el medio ambiente natural y cultural, el respeto a la diversidad, establecer un equilibrio entre las necesidades colectivas e individuales y animar a la participación de los ciudadanos. En este sentido, podemos citar los esfuerzos realizados por los Estados Unidos para tratar de paliar los males provocados por el "urban sprawl" (desparramamiento urbano), tanto en los suburbios residenciales como en la recuperación de importantes áreas degradadas en el interior de las ciudades, bajo la fórmula del "smart growth" (crecimiento inteligente) que recoge los principios del desarrollo sostenible y que supone una revisión crítica de su concepción urbanística tradicional.

Las premisas básicas sobre las que se asienta esta sostenibilidad de las ciudades son: $1^{\circ}$ ) los recursos se han de utilizar de modo eficiente, sin superar el ritmo de renovación de los recursos renovables y sustituyendo en la medida de lo posible los no renovables; $2^{\circ}$ ) el funcionamiento de la ciudad no ha de poner en peligro la salud de las personas ni superar la capacidad de carga del medio ambiente; $3^{\circ}$ ) la biodiversidad ha de ser valorada y protegida; $4^{\circ}$ ) la ciudad ha de potenciar la mezcla de funciones y la vida de barrio; y $5^{\circ}$ ) siempre que sea posible, debe intentar reducirse la dependencia del exterior, satisfaciendo las necesidades con recursos próximos. Todo un programa que cuestiona la validez de las soluciones tradicionales de los gobiernos de las ciudades y busca un escenario distinto para la ciudad, contemplada como parte de un territorio o sistema regional más amplio, introduciendo a su vez variables ambientales en los planes urbanísticos, territoriales, estratégicos y sectoriales que atañen al "buen gobierno en la ciudad sostenible". En todo caso, "la noción de sostenibilidad es dinámica y evolucionará a medida que la comprensión del medio ambiente mundial y local sea mayor y esté plenamente compartida" (COMISIÓN EUROPEA, 1996).

Aunque para la Unión Europea este debate ya había comenzado a principios de la década de los 80 con la Campaña Vivir mejor en las ciudades, no será hasta principios de la década siguiente cuando se asista al verdadero despegue en la investigación relacionada con el desarrollo sostenible. Se abría un debate a este propósito con la aparición, en un primer momento, del Libro Verde sobre el Medio Ambiente Urbano, en 1990, y la posterior aprobación del V Programa Marco sobre medio ambiente y desarrollo sostenible: Hacia la Sostenibilidad con el Proyecto Ciudades Sostenibles Europeas, iniciado en 1993, la creación de un Grupo de Expertos en Medio Ambiente Urbano y la posterior publicación 
por parte de este grupo de varios informes entre los que destaca el titulado Ciudades Europeas Sostenibles (COMISIÓN EUROPEA, 1996). Junto a otras instituciones internacionales como las Naciones Unidas se han celebrado numerosas Conferencias como la de HÁBITAT II en Estambul (1996), Madrid (CONFERENCE, 1998, que relata la aplicación de indicadores de sostenibilidad en ciudades europeas occidentales y post-socialistas) o Hannover (2000), además de sucesivas campañas del Consejo del Europa como la de "Ciudades para Vivir" y Comunicaciones que encauzan su tratamiento y aplicación. Destaca la titulada "Marco de Actuación para el Desarrollo Urbano Sostenible en la Unión Europea", en 1998, con los objetivos de acrecentar la prosperidad y el empleo, fomentar de la igualdad, la integración social y la regeneración en las zonas urbanas, proteger y mejorar el medio ambiente urbano y contribuir al buen gobierno urbano, todos ellos objetivos desarrollados a través de medidas y en programas como el Urban Sustainability and Governance in Europa (USAGE). Una de las principales actuaciones acometidas por la Unión Europea ha sido la puesta en marcha del proyecto European Sustainable Spatial Development (ESDI), el cual se empezó a gestar a fínales de la década de los 80 y que no se concretó hasta su ratificación en Postdam, en 1999. Más conocida ha sido a lo largo de los últimos años la puesta a punto de la Agenda Local 21, instrumento elegido para identificar, evaluar y difundir las propuestas innovadoras de gobierno urbano y desarrollar así la ciudad sostenible. Sucesivos proyectospiloto han desarrollado una nueva base de datos sobre gestión urbana y sostenibilidad y se han sentado las bases de buenas prácticas locales.

En este sentido, en el espacio urbano europeo preocupa el problema del deterioro de la calidad de vida y la calidad ambiental planteando ahora desde la nueva visión alternativa de la ciudad sostenible (green city, ecopolis), de la que se derivan respuestas políticas del tipo de estrategias de desarrollo urbano sostenible, la incorporación al planeamiento urbano de la protección de las tierras agrícolas y corredores ecológicos, el control medioambiental a través de la normativa de Evaluación de Impacto Ambiental, o el tratamiento de los espacios públicos. Una nueva actitud, un nuevo discurso, se está incorporando en los últimos años en la Administración Pública europea a la hora de la resolución de los problemas urbanos y de las prioridades de su resolución, lo que revisa todo el proceso desde el mismo diagnóstico e indicadores de la calidad de vida hasta el gobierno de la ciudad y su entorno.

No obstante, es preciso recordar que otros conceptos y visiones anteriores a 
los de la sostenibilidad, como los de bienestar/malestar social y calidad de vida en la ciudad, habían modificado ya los esquemas de análisis y de políticas en la escala urbana, y enlazan ahora con los derivados de la sostenibilidad, que acaparan en buena medida tanto la atención de los investigadores como de los gestores de la ciudad, teniendo como raíz la crisis urbana y ambiental actual como consecuencia del crecimiento de la ciudad capitalista y la generación de cuantiosos problemas urbanos, en estrecha asociación con las condiciones ecológicas y económicas globales, los diferentes ritmos de urbanización y talla de las ciudades, y las diferentes políticas de planeamiento y control urbano. De este modo, en unas ciudades los problemas se focalizan en el centro urbano, en otras en las periferias y suburbios; en unas ciudades significa una disminución de la población, del empleo y la producción, en otras más bien son problemas de crecimiento y ajustes. En todo caso, los problemas urbanos son bien complejos, de tipo social, económico y ambiental y alcanzan a problemas de vivienda, de tráfico y accesibilidad, de infraestructuras y dotaciones, de empleo, de segregación socioespacial, de delincuencia, institucionales y presupuestarios, de contaminación.

En los últimos años se ha asistido a la proliferación de estudios que nos llevan a medir, de una manera más segura y objetiva el desarrollo sostenible en sus múltiples aspectos. A pesar de las dificultades encontradas y de las deficiencias que aún presentan los indicadores (incertidumbre científica para los físicos y no tener en cuenta la pobreza, los recursos humanos, la riqueza y el valor del medio ambiente, entre otros, para los económicos) existen ya algunas herramientas para lograrlo, generalizándose la creación de las llamadas baterías de indicadores. Los estudios de calidad de vida se suman a los de los problemas urbanos y se profundiza en nuevos indicadores ambientales y socioeconómicos en términos del bienestar o del malestar del ciudadano. La calidad de vida es un concepto multidimensional y con alto grado de subjetividad que va más allá de la condición física o salud, incluyendo aspectos psicológicos y socioeconómicos, basados en las percepciones y expectativas de los individuos, lo que dificulta su posible medición ya que, al contrario de lo que sucede con los indicadores ambientales, para contabilizar y evaluar las cuestiones de carácter social es necesario aceptar un cierto grado de subjetividad dependiendo en gran medida de lugar al que se refiere el estudio. Los indicadores urbanos permitirán realizar un seguimiento permanente de la realidad local en el tiempo, con capacidad de analizar cada ámbito geográfico, apuntar las tendencias y permitir un alto grado 
de comparabilidad, abarcando bloques temáticos distintos de características demográficas, económicas, sociales, culturales y ambientales que conjugados pueden servir a la vez de "indicadores de sostenibilidad", tal como los que recoge el modelo de fichas de la Agenda Local 21: indicadores de habitabilidad y territorio urbano, transportes, energía, recursos hídricos, contaminaciones atmosférica y acústica, residuos, agricultura, biodiversidad y espacios naturales. Así pues, no faltan instrumentos prácticos para la aplicación de la idea de sostenibilidad, aunque en ocasiones se aprecia la falta de voluntad y decisión política para llevarla a cabo.

\section{LOS EFECTOS DE LA CIUDAD EN SU ENTORNO.}

Lo que se deduce de lo anterior es que a los viejos problemas urbanos se añaden otros derivados de la expansión de las periferias, de los nuevos suburbios, que parten de modelos de acondicionamiento residencial que utilizan tipos de urbanización de baja densidad, bien en bloques o, principalmente, a base de urbanizaciones de casas unifamiliares, rodeadas de espacios libres, que en su búsqueda del alejamiento de la congestión de la ciudad y unos parajes más naturales han provocado un incremento del transporte sin precedentes, especialmente del automóvil privado en contra de un transporte colectivo incapaz de funcionar eficazmente con unas densidades de población tan bajas. A esto se le une también la reducción de población en el centro de la ciudad y el declive y marginación de muchos de los barrios periurbanos. La inquietud por el medio natural en las áreas urbanas y metropolitanas ha conducido a un nuevo movimiento ambientalista entre los urbanistas y los representantes políticos. En la Unión Europea y países desarrollados y en menor medida en los menos desarrollados esta inquietud por el medio natural en las ciudades y sus entornos ha impulsado la adopción de políticas medioambientales a todos los niveles de gobierno.

Las periferias urbanas tienen particularmente una serie de problemas como los de la especulación del suelo que afecta en especial al suelo no urbanizable, problemas de accesibilidad, desajustes en los términos administrativos municipales y vacío de gestión supramunicipal, localización de infraestructuras altamente consumidoras de espacio o a menudo la ubicación de vertederos, abandono de la agricultura y la ganadería, etc. "La actividad económica genera externalidades negativas ambientales de polución, destrucción de hábitats y no cuenta ni con el futuro ni con la conservación de los recursos naturales escasos" (QUEIRÓS, 1998: 131). 
La situación de las ciudades de la Comunidad de Castilla y León puede ser definida según estos rasgos: la evidente ralentización del crecimiento demográfico urbano mientras se produce un fuerte proceso de extensión urbana; frente a esto, el agravamiento del declive del rico patrimonio edificado y de las grandes periferias, mal estructuradas y equipadas; y por último, las principales inversiones están destinadas a grandes equipamientos y a la recalificación del paisaje de los espacios públicos más céntricos, mientras se produce la degradación de las zonas periféricas construidas en los sesenta y setenta, por el contrario, las nuevas áreas urbanas de marcado carácter suburbano desequilibran las relaciones entre la ciudad y su entorno más inmediato. En este aspecto, se pueden sintetizar algunos de los problemas de los entornos de las áreas urbanas de Castilla y León (LÓPEZ TRIGAL, 1998) como resultado del conflicto entre la ciudad central y la ciudad de la periferia. Se trata de efectos inducidos de tipo negativo en los ámbitos de la primera y de la segunda aureola periurbana, que se pueden ejemplificar según las ciudades y distinguir de este modo:

$\left.1^{\circ}\right)$ En relación con el medio físico: geometrización y ajardinamiento de riberas fluviales, desecación y urbanización de humedales y lagunas, contaminación ambiental por escombreras e industrias, contaminación de los cursos fluviales y acuíferos en zonas de aguas debajo de la ciudad, difusión de puntos de vertidos y de escombros incontrolados, incremento de riesgo de inundaciones por avenidas, extracción y transformación de áridos en cauces fluviales, impactos visuales en el paisaje y minusvaloración del mismo.

$2^{\circ}$ ) En relación con el medio biótico: explotación abusiva por talas de la masa de vegetación, ocupación de dehesas, pinares y vegas por actuaciones urbanísticas con la consiguiente pérdida de suelo fértil, riego de cultivos de huerta con aguas sucias canalizadas.

$3^{\circ}$ ) En relación con el medio socioeconómico: abandono de los aprovechamientos agrarios y ganaderos y la sucesiva extensión de eriales, cambios de uso tradicional de suelo agrícola por usos residenciales, industriales o terciarios, localización industrial en bordes de las lindes de los términos de los municipios periurbanos, incremento y exportación de los cementerios de coches hacia la periferia, difusión de grandes instalaciones terciarias en zonas cada más distantes de la ciudad, carencias en equipamientos e infraestructuras acordes con las necesidades crecientes de los núcleos periurbanos.

$4^{\circ}$ ) En relación con el patrimonio cultural: pérdidas de patrimonio popular 
tradicional en el caserío, deterioro y pérdidas de patrimonio diseminado tal como bodegas, palomares, molinos, canales de riego, ermitas o cañadas.

$\left.5^{\circ}\right)$ Asimismo, con la organización y gobierno del territorio: ausencia de planeamiento urbanístico, de directrices territoriales (en tramitación por el momento) y de órganos de gestión supramunicipal del área urbana, ausencia incluso en algunos municipios periurbanos de una propia normativa urbanística.

Tabla 1. Evolución de la población en las ciudades de Castilla y León y sus periferias ${ }^{*}$

\begin{tabular}{|c|c|c|c|c|c|c|c|}
\hline & 1950 & 1960 & 1970 & 1981 & 1991 & 2001 & $1950-2001(\%)$ \\
\hline Ávila & 22.577 & 26.807 & 30.983 & 41.735 & 49.868 & 47.967 & 212,5 \\
\hline T.á.u.s.c. ${ }^{(* *)}$ & 2.846 & 2.720 & 1.898 & 1.707 & 1.422 & 1.421 & 49,9 \\
\hline Burgos & 74.063 & 82.177 & 119.915 & 156.449 & 169.111 & 166.251 & 224,5 \\
\hline T.á.u.s.c. ${ }^{(* *)}$ & 5.465 & 5.281 & 4.420 & 3.549 & 3.494 & 4.747 & 86,9 \\
\hline León & 59.549 & 73.483 & 105.235 & 131.134 & 147.625 & 137.384 & 230,7 \\
\hline T.á.u.s.c. ${ }^{(* *)}$ & 33.603 & 36.506 & 36.620 & 39.065 & 44.139 & 52.836 & 157,2 \\
\hline Palencia & 41.769 & 48.216 & 58.370 & 74.080 & 81.988 & 80.836 & 193,5 \\
\hline T.á.u.s.c. ${ }^{(* *)}$ & 15.184 & 17.478 & 14.638 & 14.440 & 16.356 & 16.375 & 107,8 \\
\hline Salamanca & 80.239 & 90.498 & 125.220 & 167.131 & 186.322 & 158.523 & 197,6 \\
\hline T.á.u.s.c. ${ }^{(* *)}$ & 7.022 & 8.277 & 9.066 & 9.538 & 15.117 & 27.444 & 390,8 \\
\hline Segovia & 29.568 & 33.360 & 41.880 & 53.237 & 57.617 & 54.039 & 182,8 \\
\hline T.á.u.s.c. ${ }^{(* *)}$ & 9.473 & 10.178 & 8.437 & 8.334 & 9.358 & 11.164 & 117,9 \\
\hline Soria & 16.878 & 19.301 & 25.030 & 32.039 & 35.540 & 34.640 & 205,2 \\
\hline T.á.u.s.c. ${ }^{(* *)}$ & 1.142 & 1.140 & 1.988 & 1.470 & 1.110 & 1.749 & 153,2 \\
\hline Valladolid & 124.212 & 151.807 & 236.341 & 330.242 & 345.891 & 318.293 & 256,2 \\
\hline T.á.u.s.c. ${ }^{(* *)}$ & 20.321 & 22.743 & 21.717 & 24.286 & 32.696 & 52.491 & 258,3 \\
\hline Zamora & 38.320 & 42.060 & 49.029 & 59.734 & 68.202 & 65.633 & 171,3 \\
\hline T.á.u.s.c. ${ }^{(* *)}$ & 9.500 & 9.432 & 8.522 & 7.569 & 7.490 & 8.300 & 87,4 \\
\hline Ponferrada & 23.773 & 37.053 & 45.257 & 52.499 & 59.702 & 63.233 & 266,0 \\
\hline T.á.u.s.c. ${ }^{(* *)}$ & 21.648 & 22.313 & 19.977 & 18.592 & 20.303 & 20.208 & 93,3 \\
\hline Aranda de Duero & 10.393 & 13.454 & 18.369 & 27.598 & 29.446 & 29.999 & 288,6 \\
\hline T.á.u.s.c. ${ }^{(* *)}$ & 2.547 & 2.495 & 2.086 & 1.873 & 1.623 & 1.806 & 70,9 \\
\hline Miranda de Ebro & 18.094 & 27.881 & 33.905 & 36.812 & 37.197 & 35.470 & 196,0 \\
\hline
\end{tabular}

(*) Establecidas por la Junta de Castilla y León en sus Directrices de Ordenación del Territorio, pp. 52 y 55

${ }^{(* *)}$ Total área urbana sin capital

FUENTE: INE. Censos de Población. 
El balance entre los espacios periféricos y los espacios centrales y pericentrales de la ciudad está así a menudo inserto en una situación de conflicto y tensión, en el que juega cada vez una mayor ventaja la periferia, pues el desplazamiento del equilibrio demográfico y económico del centro hacia la periferia ya ha comenzado (Zamora, Ponferrada, Burgos) o consolidado (Salamanca, Valladolid y León) según los casos y parece irreversible. Un repaso a la Tabla 1 nos muestra las tendencias de crecimiento de la población en las capitales provinciales y sus áreas más inmediatas que han sido clasificadas por el Documento de avance de Directrices de Ordenación del Territorio de Castilla y León (DOT) como "nodos urbanos".

En el futuro se ganarán las elecciones en las periferias y el ritmo de producción inmobiliaria se hará mayor en ellas que en los barrios periféricos de la ciudad. La cuestión es, desde el punto de vista de una fuerte carga de sostenibilidad, equilibrar economía y medio ambiente, imponiendo límites a las actividades económicas y humanas que afectan al medio natural, frenar el proceso de "desparramamiento" de nuestras ciudades, controlar los numerosos efectos negativos y potenciar a su vez los efectos positivos sobre el entorno, sin olvidar el evitar el declive de los espacios centrales y pericentrales. "A la luz de este marco ideológico-político la calidad de vida urbana es un producto de decisiones políticas enfocadas por los objetivos económicos y cercadas por la preservación ambiental. Es un proceso que afecta y envuelve a todos los ciudadanos al hacer presente que no se pueden negar a las generaciones futuras los recursos que precisarán" (QUEIRÓS, 1998: 138).

\section{PRÁCTICAS DEL NUEVO URBANISMO SOSTENIBLE.}

Este panorama exige intervenciones, que se han demorado desde hace tiempo en las ciudades con el ánimo de abordar decididamente la recualificación y el ámbito de lo "social-urbano" de los barrios desfavorecidos y del centro de la ciudad al mismo tiempo que actuaciones dirigidas a la coordinación y la cooperación intermunicipal, sea de tipo obligatorio o sea de tipo facultativo, en lo que llamaríamos el "espacio de la negociación y del consenso" de las periferias urbanas. "Sin embargo, en este debate hay que recordar dos cosas. En primer lugar, la acción del urbanista no resolverá todos los problemas de las periferias. Las tragedias de la marginación, del paro de larga duración, de la exclusión de ciertas comunidades, no van a desaparecer de un día para otro sólo porque el entorno construido sea más hermoso y más amable... En segundo lugar, la ciu- 
dad es el reino de la larga duración, del largo plazo...Es como decir que las decisiones de fondo van mucho más allá que los plazos electorales". Aunque el mismo autor más adelante señala que "no obstante, los problemas de las periferias calientes han adquirido tal gravedad... en la lucha contra la inseguridad, contra la marginación... que las mejoras se tornarán una prioridad" (BOFILL, 1998: 116).

Cabe presentar ciertas prácticas en relación con el urbanismo sostenible y las periferias urbanas, entrevistas desde la interdependencia de los problemas ambientales y socioeconómicos, los límites del crecimiento y el proceso de urbanización, partiendo, según el V Programa Marco de la Unión Europea, de que en el medio urbano, primero, la fuerza actuante es la urbanización congestiva y contaminante, segundo, su efecto negativo es la pérdida de calidad de vida y entorno y, tercero, el objetivo es la mejora en el desarrollo urbano, esto es, la calidad medioambiental, la racionalidad de la estructura espacial urbanística, la cohesión de la sociedad y el desarrollo local que minimiza los impactos ambientales.

Las estrategias alternativas de este nuevo urbanismo parten ante todo de un movimiento medioambiental que es consciente de que los procesos ecológicos que operan en la ciudad forman una base indispensable para restaurar el paisaje urbano a partir de las interrelaciones y la interdependencia entre los elementos del medio físico. Desde esta premisa, en las ciudades hace falta una mayor valoración, por ejemplo, de "los hábitats potenciales para plantas y animales fragmentos de bosque o prados, valles y otros conectores - que deberían ser integrados en la planificación de la red social urbana" (HOUGH, 1998: 110). Este mismo autor propuso en 1987 un sistema de clasificación medioambiental para los espacios urbanos, a partir de su experiencia planificadora en Toronto, que contempla para cinco escalas de parques, por un lado, la disminución de los niveles de mantenimiento e intensidad de uso, y por otro, el incremento de los valores medioambientales y sensibilidad a la perturbación, a medida que se considera un mayor tamaño del parque.

Esta política de restauración ecológica se puede llevar a cabo a la hora de la restauración de un parque urbano en los espacios centrales o pericentrales (la "ciudad arbolada") como en un programa de naturalización para un sistema de parques urbanos en la periferia. Este segundo supuesto ha sido llevado a cabo en ciudades como Zurich con la gestión integrada de parques forestales que a la vez que se mantienen económica y ecológicamente autosostenibles rebate la 
idea de que los parques son exclusivamente para el recreo. Curitiba, ciudad brasileña caracterizada por el desarrollo con calidad de vida y en especial en el transporte colectivo, mantiene asimismo prácticas de ecología urbana y de respeto al medio ambiente en el planeamiento urbano que van desde líneas de formación en este campo al mantenimiento de grandes parques urbanos. O la ciudad de Porto, que en los últimos años ha diseñado el nuevo "Parque da Cidade", de unas 90 Hectáreas, que permitirá un reequilibrio de la ciudad desde las exigencias ambientales, y resultado de un sutil trabajo de ingeniería y arquitectura paisajística donde hasta los lagos son alimentados por las aguas de lluvia, en contrapunto con los espacios desarbolados o densamente urbanizados de gran parte de la ciudad tradicional.

Dentro de los casos españoles podemos hablar de las experiencias recogidas en el libro "Ecourbanismo, Entornos Humanos Sostenibles: 60 proyectos" (RUANO, 1999), algunas de ellas ya construidas y otras todavía en proyecto. En el proyecto Can Llobera, en San Feliu de Llobregat se aborda la construcción de un nuevo barrio en el centro de la localidad de reducidas dimensiones $(4,5 \mathrm{hec}-$ táreas), mientras los otros tres hacen referencia a la construcción del Parque Balear de Innovación Telemática en Mallorca, la construcción de una Ecoisla en la fachada costera de la ciudad de Barcelona o la urbanización bioclimática en la isla de Tenerife. En este ámbito español hay ya un primer balance de las prácticas urbanas de sostenibilidad (PRATS, VARIOS AUTORES, 1998-1999) en el que se llega a unas conclusiones provisionales: a) todavía no existe una conciencia arraigada sobre los problemas de la sostenibilidad urbana, b) hasta ahora la política ha carecido de la visión territorial de tipo supramunicipal y de alcance sobre los entornos rurales y naturales de las ciudades, c) la supeditación acrítica hacia el crecimiento sin límites como el factor de desarrollo, sin mediar una evaluación sobre los impactos, d) en los años noventa se inician ciertas prácticas orientadas a mejorar la habitabilidad y calidad de vida, aunque a veces se limitan a aspectos físicos y paisajísticos superficiales y quedan relegados los aspectos más sustantivos relacionados con la sostenibilidad local.

Una buena base de estudio de la evolución de las buenas prácticas en materia de sostenibilidad la tenemos en las listas de experiencias premiadas y nominadas en el Concurso de Buenas Prácticas, que con carácter bianual, patrocina el Emirato de Dubai (http://habitat.aq.upm.es). En el caso español, la participación en estos premios ha ido en aumento, en las ediciones celebradas (1996, 1998 y 2000) se han presentado un total de 114 prácticas (44, 35 y 35 respectivamente) 
siendo premiadas un total de 74 ( 9,33 y 32). Su distribución espacial y los temas abordados podemos observarlos en las siguientes tablas.

Tabla 2: Prácticas premiadas y nominadas según C.C.A.A.

\begin{tabular}{lc}
\hline C.C.A.A. & $\mathrm{N}^{\circ}$ buenas prácticas \\
\hline Andalucía & 18 \\
Aragón & 3 \\
Asturias & 3 \\
Cantabria & 2 \\
Castilla y León & 6 \\
Cataluña & 19 \\
Extremadura & 1 \\
Galicia & 4 \\
Islas Baleares & 8 \\
Islas Canarias & 2 \\
Madrid & 17 \\
Murcia & 1 \\
Navarra & 5 \\
País Vasco & 7 \\
Comunidad Valenciana & 5 \\
España & 13 \\
\hline Total & 114 \\
\hline
\end{tabular}

FUENTE: http://habitat.aq.upm

Tabla 3: Prácticas premiadas y nominadas según tema

\begin{tabular}{lc}
\hline Tema & $\mathrm{N}^{\circ}$ prácticas \\
\hline Urbanismo & 26 \\
Vivienda & 11 \\
Participación ciudadana & 11 \\
Protección áreas sensibles & 10 \\
Acción social & 7 \\
Zonas verdes & 6 \\
Transporte & 5 \\
Residuos & 5 \\
Aguas & 5 \\
Otros & 28 \\
\hline Total & 114 \\
\hline
\end{tabular}

FUENTE: http://habitat.aq.upm 
En primer lugar, el inicio de estas prácticas arranca de la necesidad de innovar los modelos territoriales y los modelos urbanos y metropolitanos, a partir de nuevas normativas como las Directrices de ámbito regional y de áreas urbanas, que podemos ejemplificar entre otras en el Plan de Ordenación del Territorio de Andalucía (1999) en cuyas estrategias para el sistema de ciudades formula "criterios para una gestión sostenible del medio ambiente urbano como factor esencial para la calidad de vida en las ciudades y la mejora de su balance ecológico global". En paralelo, desde finales de los años ochenta se han venido gestando por los municipios urbanos Planes Estratégicos, que en la última generación han recogido programas y actuaciones de sostenibilidad en algunos de ellos, o bien en el mismo planeamiento urbano general o especial se ha incidido en programas de desarrollo urbano integrado, de lucha contra la exclusión social, de protección del entorno natural y de frentes fluviales y marítimos, de peatonalización, de gestión ambiental de los residuos urbanos y de la industria, de impulso del transporte colectivo e introducción del transporte no contaminante.

Los casos de ciudades españolas donde se aplican programas de sostenibilidad en diferentes áreas (entornos, residuos, transportes, desarrollo urbano...) no son tantos como ya se ha podido comprobar en las tablas anteriores, pues "el adjetivo sostenible, como epíteto prestigioso y poco comprometido, se derrumba en cuanto analizamos la sostenibilidad, como sustantivo con contenidos precisos en forma de limitaciones físicas y ecológicas... sólo la convergencia más o menos sinérgica de acciones confluyentes puede hacernos señalar como adecuadas ciertas ciudades. Es el caso de Vitoria y de Girona, por citar dos casos; pero ambos son también exponentes de buenas situaciones heredadas de un pasado inmediato no demasiado degradante o duro" (PARRA, VARIOS AUTORES, 1998-1999).

En Vitoria, como decíamos anteriormente, ciudad compacta pero no congestiva y bien planificada desde hace décadas, los programas se han detenido especialmente en los campos de la rehabilitación integral de su pequeño casco antiguo, del bienestar social y calidad de vida de los barrios periféricos, de la accesibilidad intraurbana y periférica, de la peatonalización y el transporte colectivo, de la gestión del ciclo del agua, de la densificación de zonas verdes, etc. por todo ello la evaluación de los indicadores de sostenibilidad son de los más altos de las ciudades españolas. Mientras que Girona, que había asumido en los años ochenta políticas urbanísticas que incrementaron la calidad de vida de sus ciudadanos, en los años noventa se ha impulsado y gestionado desde el plan estra- 
tégico ciertas mejoras que parten de un amplio proceso de participación ciudadana y se dirigen entre otras medidas al cuidado de su entorno y del medio ambiente y el paisaje urbano y las mejoras del desarrollo y la movilidad.

Otras ciudades han llevado a cabo también programas en sus periferias urbanas a fin de mejorar asentamientos de infravivienda (Plan de Remodelación de Barrios de Madrid) en colaboración entre los residentes y la Administración o han puesto en acción programas de diversa índole que enlazan ahora con los de la Agenda Local 21. El escaso interés de ciertos grupos políticos por esta temática ha provocado situaciones extrañas como la que ha sufrido la Comunidad de las Islas Baleares, donde el acceso al poder del Partido Popular ha llevado a la eliminación de ciertos proyectos y medidas relativos a la sostenibilidad de las islas que el gobierno anterior había llevado a cabo, como la disolución del Consejo Asesor por la Sostenibilidad de las Islas Baleares y cuyo ejemplo más conocido es el de la ecotasa (pago de $1 €$ por día de estancia turística, destinado al mantenimiento, protección y conservación de su medio ambiente).

\section{PRÁCTICAS INICIALES DE SOSTENIBILIDAD EN CASTILLA Y LEÓN.}

Como explicación previa debemos tener en consideración que muchas de las actividades o proyectos etiquetados como sostenibles no lo son en absoluto, mientras que otros que no usan ese adjetivo pueden incluirse dentro. Entre estas últimas podemos citar algunas experiencias, aún en una fase inicial, que van desde estudios de la contaminación acústica (mapas del ruido de León, Ávila, Palencia, Salamanca, Segovia, Zamora...) a algunos planes y programas. Las circunstancias son diferentes para las Agendas Locales 21, instrumento clave desarrollado por la Cumbre de Río, aunque en estos casos permanecen en distintos grados de aplicación y sostenibilidad, entre otras ciudades Valladolid, Burgos o Aranda de Duero.

En la ciudad de León, se llevó a cabo en 1995 un primer Mapa Acústico que ha sido completado por un segundo y que ha supuesto el estudio de la medición del ruido en varios puntos y a diferentes horas y cuyos objetivos se encaminan al control de ruidos de tráfico e instalaciones de hostelería así como a la planificación urbana. Otras prácticas han sido puestas a punto por programas de escuelas taller para la mejora de fachadas de edificios, de peatonalización de sectores del centro y del casco antiguo, en materia de divulgación y formación me- 
dioambiental, de depuración del agua y de recuperación simbólica de prácticas agrícolas en terrenos ribereños ganados con la canalización de los dos ríos locales (el "Coto Municipal Escolar" y los "Huertos de la Candamia" se han convertido en las dos prácticas más singulares de sostenibilidad). Mientras tanto, la otra cara de la moneda en León, al igual que en algunas otras ciudades de la región, es la desatención de momento al tratamiento de residuos sólidos, la creación de nuevos parques urbanos nada sostenibles, la congestión del tráfico en ciertos tramos y una mala accesibilidad general, el deterioro de buena parte del casco antiguo, arrabales y barrios periféricos, la permanencia de vacíos industriales, así como una suburbanización sin suficiente control y que ocupa las vegas del entorno agrario y deja en barbecho amplios eriales.

Siguiendo con el caso de la ciudad de León, los últimos cambios se aprecian, de un lado, en la creación del nuevo paseo fluvial del Bernesga, en su tramo Norte, que incorpora numerosas novedades de diseño no utilizadas anteriormente en el tratamiento de otros sectores. Así vemos como frente a la geometrización se ha buscado un cauce más sinuoso, creando isletas a lo largo del parque, como refugios de los ecosistemas fluviales y un mayor acercamiento de los paseantes a las perspectivas del río. Por otro lado, en 1992 se iniciaba la revisión del Plan General de Ordenación Urbana, llegándose a publicar en 1996 un documento de Avance que recogía la práctica totalidad de los postulados del desarrollo sostenible, quedando abandonado en el olvido en los reajustes posteriores hasta el punto que en 2002 se presenta un nuevo documento para la aprobación inicial con radicales diferencias, pues se caracterizaba por ser altamente especulativo y retrógrado (zonificación y ladrillo sin piedad) lo que empuja a mantener una mayor vigilancia en aquellos aspectos donde los intereses políticos y económicos desplazan al interés general. La misma empresa recibe recientemente el encargo de reajustar por tercera vez el documento, a partir de una distinta representación de las fuerzas políticas desde 2003.

En otras ciudades de Castilla y León se pueden seleccionar prácticas de distinta envergadura y contenido con resultados dispares. Primeramente, en Segovia se encuentra la más consistente experiencia en este campo, el "Plan Verde para una Ciudad Patrimonio de la Humanidad", que se inicia en 1991 con un movimiento ciudadano concienciado por el mal estado de los cauces fluviales del Eresma y del Clamores que abrazan la ciudad y que se pretenden recuperar con proyectos de accesos y movilidad, de corredores paisajísticos, de reforestación y saneamiento integral. En la localidad segoviana de Cuellar se ha cons- 
truido una planta de calefacción y agua caliente sanitaria que permite el uso de los residuos de biomasa procedentes de la limpieza de la importante mancha forestal que la rodea. En la ciudad de Salamanca, en su arrabal allende el río Tormes, hoy transformado en las barriadas de Buenos Aires, Tejares, Chamberí, Zurguen y San José, ha dado comienzo en 1996, amparado en la Iniciativa de Empleo HORIZON, un Programa de "Desarrollo Comunitario e Intervención Sociolaboral en la Periferia de la Ciudad" que atiende, aunque con un escaso presupuesto, a personas de clase social baja, en parte de etnia gitana, marginadas tradicionalmente, a fin de generar empleo, impulsar la participación y la convivencia ciudadana y planes de mejora en materia de salud, prevención de la drogadicción, formación y medio ambiente. En la ciudad de Valladolid, los esfuerzos se han destinado a la mejora de los barrios periféricos de la ciudad, especialmente centrado en los Barrios España y San Pedro Regalado o el desarrollado en el Poblado de La Esperanza dirigido, entre otras cosas, al realojo de familias y su integración social. También se han realizado estudios en el Barrio de La Rondilla con objetivos similares. Otro elemento significativo ha sido el realizado en la provincia de Soria que cuenta con el mayor grado de aceptación de las Agendas 21 Locales gracias a la ONG DEYNA.

En suma, el nuevo modelo de ciudad difusa, traducido en términos de área metropolitana o área urbana y por tanto de expansión sin límites en la periferia o entorno, alcanza ya a la mayor parte de las ciudades españolas y europeas de tamaño grande y mediano e incluso empieza a vislumbrarse en alguna ciudad pequeña y en núcleos semiurbanos, de ahí la necesidad perentoria de hacer balance del desarrollo y consolidación de las periferias urbanas desde indicadores y principios de sostenibilidad así como hacer frente a los problemas urbanos de las periferias a partir de la intervención, planeamiento y gestión de tipo supramunicipal. En este orden, es preciso que en Castilla y León se combinen las prácticas de sostenibilidad con todo tipo de planeamiento y en especial con las Directrices de Territoriales de las Áreas Urbanas y de Áreas Funcionales, en proceso lento de tramitación en la actualidad.

\section{BIBLIOGRAFÍA}

BofiLl, R. (1998) La ciudad del arquitecto. Barcelona: Círculo de Lectores. Conference On URban AND Regional ReSEARCH. ViII (1998) Major trends characterizing human settlements. Development in the ECE Region. Madrid: Naciones Unidas-Ministerio de Fomento. 
COMISIÓN EUROPEA (1996) Ciudades Europeas Sostenibles. Bruselas.

COMISIÓN EUROPEA (1999) Estrategia Territorial Europea. Luxemburgo: Oficina de Publicaciones Oficiales de las Comunidades Europeas.

FERRER, M. (1998) «Spain: recuperation, quality of life and sustainability within the context of HABITAT II», in C. Beguinot, A. Notarangelo, Habitat recovery for the city in the XXI century. Napoli: Universitá di Napoli.

GRUPO ADUAR (2000) Diccionario de geografía urbana, urbanismo y ordenación del territorio. Barcelona: Ariel.

Hough, M. (1998) Naturaleza y ciudad. Barcelona: Gustavo Gili.

LÓPEZ TRIGAL, L. dir. (1996) Ciudad y alfoz. Jornadas de estudio y debate urbanos. León: Universidad de León.

LÓPEZ TRIGAL, L. (1998) «Áreas urbanas y ciudades medias de Castilla y León. Rasgos, problemas y políticas». Medio Ambiente, 9, pp. 44-49.

Ministerio DE FOMENTO (2000) La desigualdad urbana en España. Madrid: Centro de Publicaciones Ministerio de Fomento.

MONCLÚS, F.J. (1998) «Suburbanización y nuevas periferias. Perspectivas geográfico-urbanísticas» en La ciudad dispersa: suburbanización y nuevas periferias. Barcelona: Centro de Cultura Contemporánea de Barcelona, pp. 5-15 NAREDO, J.M. (2000) Ciudades y crisis de civilización. Madrid: http://www.habitat.aq.upm.es

OCDE / MOPU (1991) El entorno urbano: políticas medioambientales para los años 90. Madrid.

PigeON, P. (1994) Ville et environment. Paris: Nathan.

QuEIRós, M. (1998) «O paradigma da sustentabilidade. Cambios para a substituiçao da versao fraca pela forte em áreas urbanas». Cadernos de Geografia, 17, pp. 131-138.

RUEDA, S. (2002) Barcelona, ciutat mediterrània, compacta i complexa. Una visió de futur més sostenible. Barcelona: Ajuntament de Barcelona.

REDCLIF, M. (2000) «El desarrollo sostenible: necesidades, valores y derechos» en Varios Autores, Desarrollo sostenible: un concepto polémico. Bilbao: Universidad del País Vasco, pp. 17-38

RUANO, M. (1999) Ecourbanismo, Entornos Humanos Sostenibles: 60 proyectos. Barcelona: Gustavo Gili.

TORRES I CAPELL, M. DE (1997) «La formación de un paisaje metropolitano». Obras Públicas, 39, pp. 66-75.

VARIOS AUTORES (1998-1999) Ciudades para un futuro más sostenible. Madrid: Ministerio de Fomento, http://habitat.aq.upm.es. 


\title{
RESUMEN
}

El objetivo de este trabajo es revisar conceptos y enfoques en torno a ciudad, periferia y sostenibilidad. Para ello se presentan las interrelaciones ciudad-centro, ciudad pericentral y ciudad-periferia, seguidas de las existentes entre ciudad y medio ambiente, y por otro lado, de los conceptos derivados del "nuevo urbanismo sostenible" que nos conduce a los de calidad de vida y problemas urbanos, bienestar y malestar en la ciudad a partir de la valoración de distintos indicadores. Finalmente, se ejemplifican ciertas buenas prácticas de sostenibilidad en periferias de las ciudades españolas y en particular de las castellanas y leonesas.

Palabras clave: Ciudad y sostenibilidad. Periferias urbanas. Ciudades españolas. Ciudades castellanas y leonesas.

\begin{abstract}
The objective of this work is to revise concepts and focuses around city, periphery and sustentability. For this it aims to present the interrelations city-center, pericentral city and city-periphery, followed by the existent ones between city and environment, and on the other hand, those of the derived concepts of the "new sustainable urbanism" which leads us to those of quality of life and urban problems, well-being and uneasiness in the city starting from the assessment of different indicators. Finally, certain good sostenibility practices are exemplified in peripheries of the Spanish cities and in particular of the Castille and León region.
\end{abstract}

Keywords: City and sostenibility. Urban peripheries. Spanish cities. Castilla and León cities.

\section{RÉSUMÉ}

L'objective de ce travail est de réviser des concepts e des points de vue autour de la cité, la périphérie et le développement durable. Pour cela, cet articule présente les interrelations cité-centre, cité péricentral et cité-périphérie, suivies par celles existantes entre cité et environnement, et ,dans l'autre côté, celles des concepts dérivés du "nouveau urbanisme durable" que nous conduit á ceux de qualité du vie et de problèmes urbains, bien-être et malaise dans la cité à partir de la évaluation de différents indicateurs. Finalement, on exemplifie certaines bonnes pratiques de développement durable dans les périphéries des cités espagnoles et en particulier de celles de Castille et León.

Mots clés: Cité et développement durable. Périphéries urbaines. Cités espagnoles. Cités de Castille et León. 